\title{
Remote monitoring of the earthquake cycle using satellite radar interferometry
}

\author{
By TIM J. WRIGHT \\ Department of Earth Sciences, University of Oxford, \\ Parks Road, Oxford OX1 3PR, UK
}

Published online 17 October 2002

The earthquake cycle is poorly understood. Earthquakes continue to occur on previously unrecognized faults. Earthquake prediction seems impossible. These remain the facts despite nearly 100 years of intensive study since the earthquake cycle was first conceptualized. Using data acquired from satellites in orbit $800 \mathrm{~km}$ above the Earth, a new technique, radar interferometry (InSAR), has the potential to solve these problems. For the first time, detailed maps of the warping of the Earth's surface during the earthquake cycle can be obtained with a spatial resolution of a few tens of metres and a precision of a few millimetres. InSAR does not need equipment on the ground or expensive field campaigns, so it can gather crucial data on earthquakes and the seismic cycle from some of the remotest areas of the planet. In this article, I review some of the remarkable observations of the earthquake cycle already made using radar interferometry and speculate on breakthroughs that are tantalizingly close.

Keywords: earthquake cycle; satellite radar interferometry; InSAR; geodesy

\section{The earthquake cycle}

In the small hours of 17 August 1999, the largest earthquake to hit Turkey in 60 years devastated the town of Izmit and the surrounding region. More than 18000 people lost their lives and 250000 people were made homeless. The earthquake cost the Turkish economy around $\$ 6$ billion. The Izmit earthquake, like most large continental earthquakes, literally tore the Earth - the pulse emanating from the epicentre at a speed of $11000 \mathrm{~km} \mathrm{~h}^{-1}$ ruptured a $130 \mathrm{~km}$ section of a major fault in the Earth's crust in just $40 \mathrm{~s}$. The region immediately to the south of the fault moved to the west compared with the northern side; walls, roads, railway lines and rivers that once crossed the fault in a straight line were given a new step, up to $5 \mathrm{~m}$ in magnitude (figure 1). This was not the first earthquake to have occurred in this area; historical records show that very similar events occurred in 1719 and 1894 (Ambraseys \& Jackson 2000). These earthquakes occurred on the North Anatolian Fault, an extremely active fault that accommodates the westward escape of Turkey as it is squeezed between the northward motion of the Arabian plate and the Eurasian plate, which blocks its path (figure 2). It is this repetition of earthquakes on the same fault that we refer to as the earthquake cycle.

One contribution of 20 to a Triennial Issue 'Astronomy and Earth science'. 


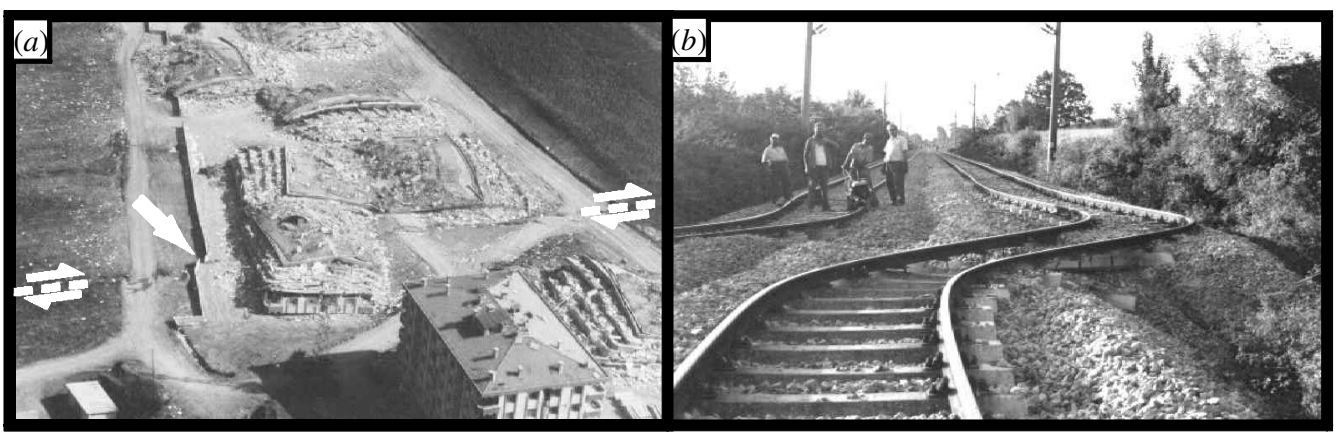

Figure 1. Surface rupture of the Izmit earthquake. (a) Newly completed accommodation blocks, unfortunately located directly on top of the North Anatolian Fault, but fortunately yet to be occupied. The surface rupture and sense of motion is shown by the dashed lines, with the large white arrow indicating the $4 \mathrm{~m}$ offset in the wall to the complex as it crosses the rupture. (b) A newly created 2-3 m step in the Istanbul to Ankara mainline railway. (Photographs courtesy of Aykut Barka, Istanbul Technical University.)

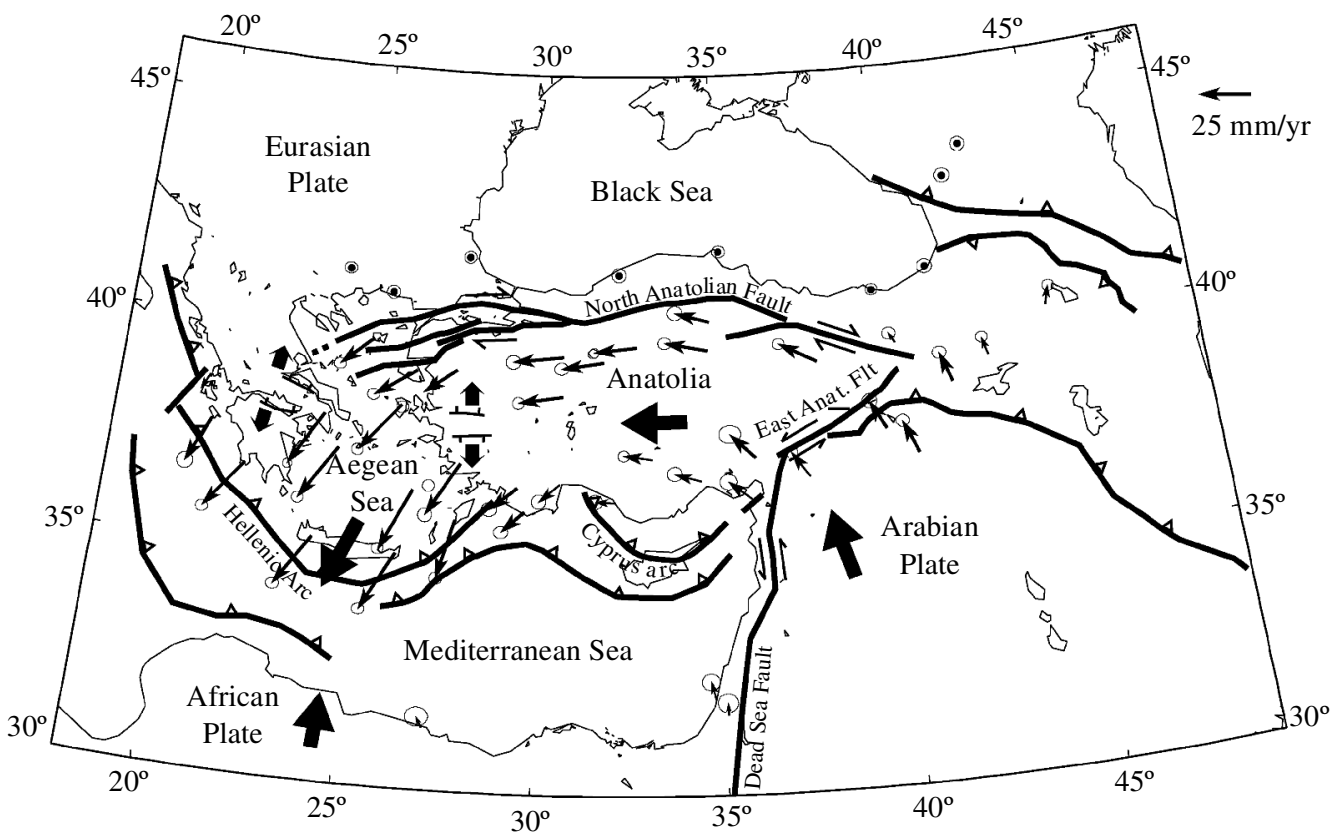

Figure 2. Tectonic setting of Turkey. Velocity (arrows) was measured by the Global Positioning System (GPS) during the interval 1988-1998 and are shown relative to Eurasia (McClusky et al. 2000). The Anatolian block is caught between the northward motion of Arabia and Eurasia and is forced westwards. The major faults are shown.

In its simplest form, the idea of the earthquake cycle was developed by Harry Fielding Reid, to explain observations of the San Francisco Earthquake of 1906, associated with an average $4-5 \mathrm{~m}$ surface slip along $450 \mathrm{~km}$ of the San Andreas Fault (Scholz 1990). Reid examined precise survey data from a triangulation network that spanned the fault. It had been carefully measured in the 1880 s and again immediately after the earthquake. For the first time, these observations revealed the surface deforma- 

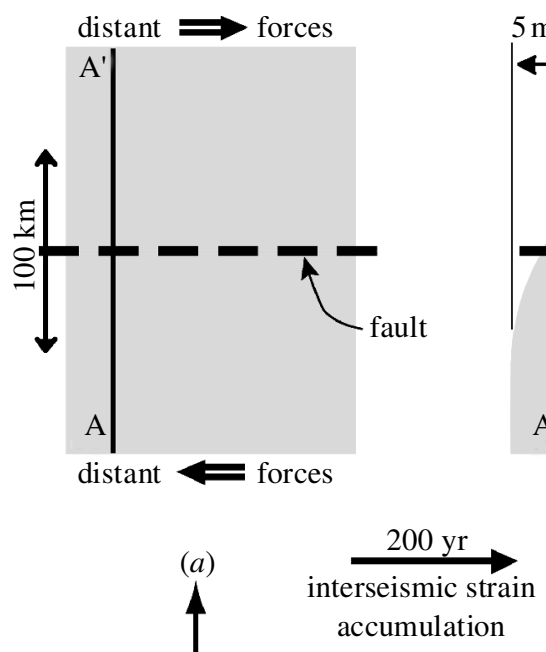
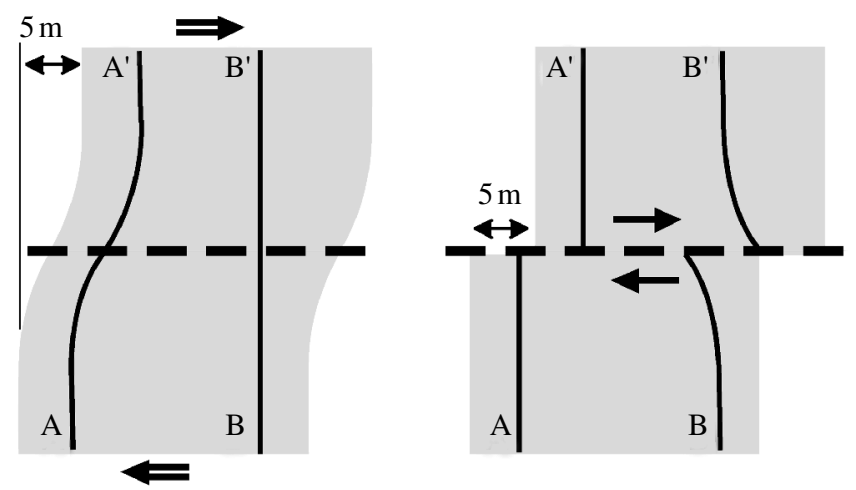

(b)

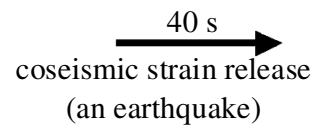

Figure 3. Schematic of Reid's elastic rebound model of the earthquake cycle. (a) Map view of area spanning a hypothetical fault, in the instant after the last earthquake. (b) The same area, 200 years later. The profile $\mathrm{A}-\mathrm{A}^{\prime}$, straight at the beginning of the cycle, has become curved. This is known as interseismic strain accumulation. Note that the magnitude of the warping is vastly exaggerated in this diagram. (c) $40 \mathrm{~s}$ later, after an earthquake. $\mathrm{A}-\mathrm{A}^{\prime}$ is once more a straight line, but this time with a $5 \mathrm{~m}$ step at the fault. B- ${ }^{\prime}$, straight immediately before the earthquake, is now curved with an offset of $5 \mathrm{~m}$ at the fault, decaying with large distances from the fault. The timings and displacements are representative of a typical earthquake, such as the 1999 Izmit event.

tion caused by an earthquake. Points on the southwest side of the San Andreas Fault had moved to the northwest during the earthquake, compared with points on the other side of the fault, and when these displacements were extrapolated onto the fault they matched the offsets observed at the ground break. Importantly, the magnitude of movement of the survey points decayed rapidly away from the fault, so that they were small at distances of $20 \mathrm{~km}$ or more.

Furthermore, on comparing survey data from the 1860s with that of the 1880 s, Reid noticed that the Farallon lighthouse, a point a long way to the southwest of the fault, had moved in a northwesterly direction in this period when compared with points on the other side of the fault. These observations led Reid to propose his elastic rebound model of the earthquake cycle (figure 3) (see also Reid 1910), in which 'distant forces' $\dagger$ gradually strain the region around the fault, building up elastic energy, typically over a period of hundreds to thousands of years, until frictional forces on the fault surface are overcome and breaking point is reached. The elastic energy is then suddenly and catastrophically released; the regions on either side of the fault rebound and the ground breaks along the fault in an earthquake. The cycle then begins again. Over long periods of time this stick-slip behaviour results in the large cumulative fault offsets observed in the geological record: around $75 \mathrm{~km}$ for the North Anatolian Fault (Armijo et al. 1999), an offset that would take something like

$\dagger$ This was before the idea of plate tectonics had been developed, so Reid's model did not suggest a driving mechanism. 


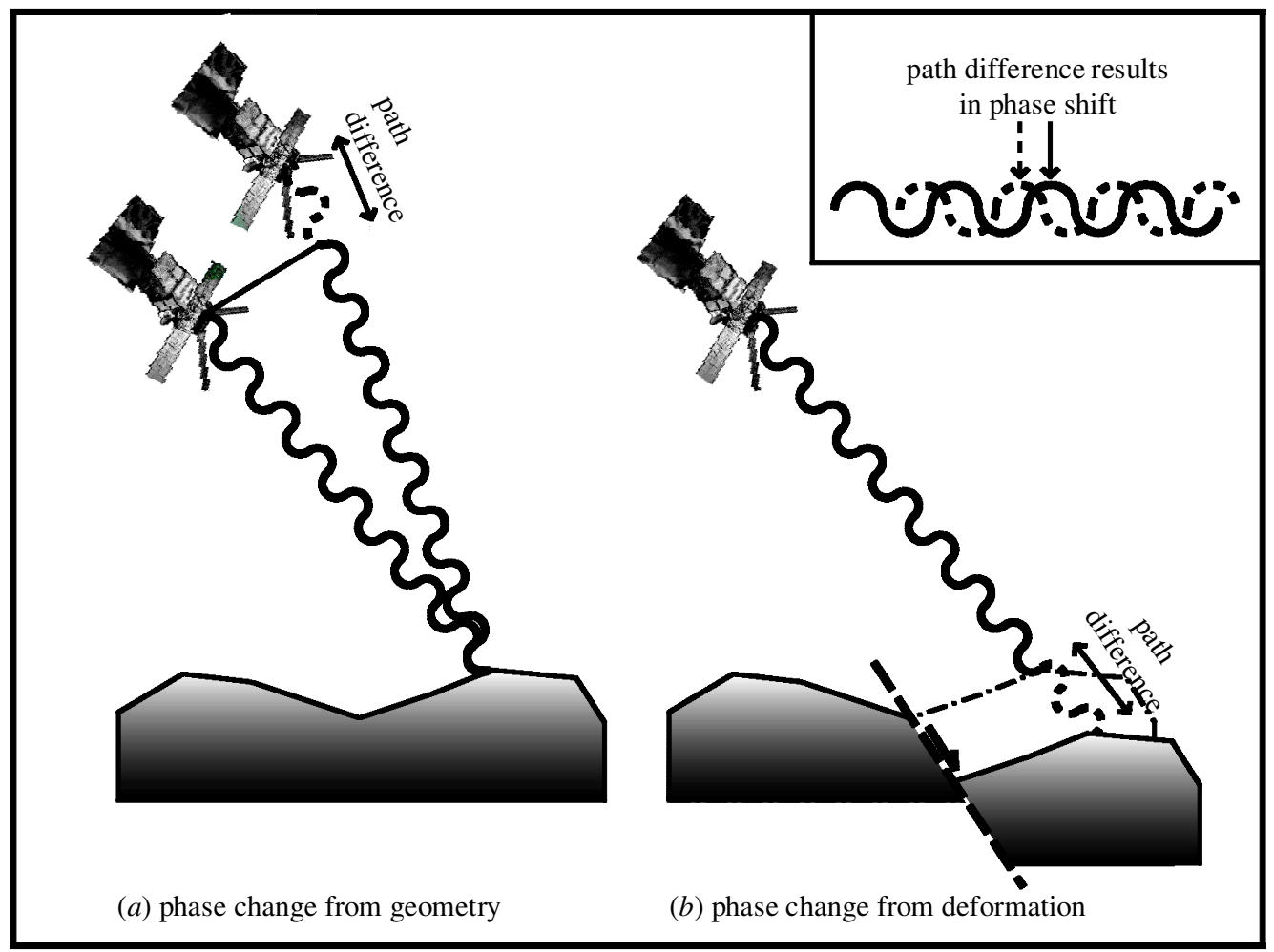

Figure 4. Schematic of radar interferometry: $(a)$ two satellites image the same point on the ground at different times but from different positions, creating a phase shift; $(b)$ a point on the ground imaged from the same point in space, before and after an earthquake. The phase change induced is directly related to the component of surface deformation in the look-direction of the satellite (the path of the radar wave). Note that this image is not to scale: ERS-1 and ERS-2 orbit at $780 \mathrm{~km}$ with a wavelength of $5.6 \mathrm{~cm}$.

15000 Izmit-like earthquakes and over three million years to accrue at present rates of deformation.

We now know that Reid's elastic-rebound model is too simplistic. Because it ignores changes in the properties of rocks with depth, it cannot explain why interseismic deformation is focused on the fault that eventually ruptures or why rapid deformation often occurs in the immediate aftermath of an earthquake (postseismic deformation). There is broad agreement that most continental earthquakes occur in the so-called seismogenic crust, typically the upper $10-20 \mathrm{~km}$, and that this behaves elastically, just as in Reid's model. Below this, where rocks are hotter, the material properties and behaviour of continental crust are still controversial. Observations of surface deformation at various stages in the earthquake cycle, many now coming from InSAR, are beginning to place bounds on competing models.

\section{Satellite radar interferometry}

The majority of today's remote-sensing satellites operate at optical wavelengths, primarily recording light originating from the Sun that makes it to the satellite's 


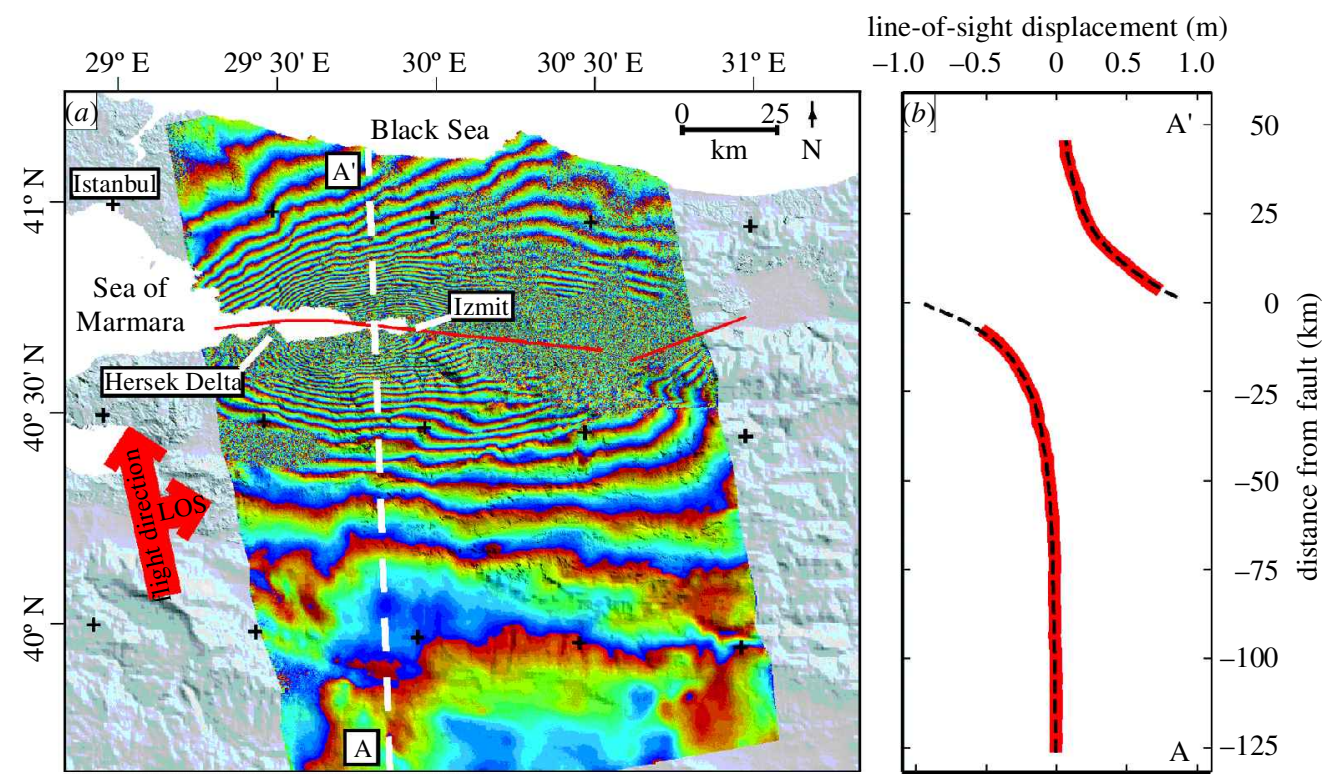

Figure 5. (a) Radar interferogram mapping the deformation field caused by the 1999 Izmit (Turkey) earthquake, the rupture of which is shown by the red line. Each coloured interference fringe is equivalent to a $28 \mathrm{~mm}$ contour of surface displacement in the satellite's line of sight (LOS; red arrow). (b) LOS displacements along profile $\mathrm{A}-\mathrm{A}^{\prime}$, revealing a characteristic elastic rebound form (red line). The dashed black line is a profile through a simple elastic model of the earthquake.

sensors, having been reflected or scattered off the Earth's surface. Radar satellites, such as the European Space Agency's Earth Resources Satellites, ERS-1 and ERS-2, are different: they actively illuminate the Earth, recording the backscattered waves and, because radar wavelengths (typically $5-25 \mathrm{~cm}$ ) are around 100000 times longer than the wavelength of visible light, radar travels through clouds. Radar satellites can therefore operate night and day and in all weather conditions.

None of the radar satellites currently in orbit was designed to measure the Earth's deformation. Nevertheless, because they are illuminating the Earth with controlled, coherent radar waves, radar interferometry is possible. In essence, interferometry works by ignoring the amplitude of the waves that return to the satellite's antenna. Instead, the phase $\dagger$ of the wave is used (figure 4). We know the wavelength of the radar waves, the phase of the waves when they left the satellite and the phase of the waves returning from a particular patch of ground (or pixel in the radar image). The distance from the satellite to the ground is simply a large unknown number of whole wavelengths (around 30 million for ERS-1 and ERS-2) plus a known fraction of that wavelength, determined from the difference in phase between the waves that leave and those that return.

This does not initially seem like a very practical method for measuring distances, particularly when we also know that there is a random phase shift added to each measurement when the wave bounces off the ground. But imagine returning to exactly

$\dagger$ The phase of a wave describes its position within the wave cycle, i.e. if it is at a peak, or trough, or somewhere in between. 
the same position in space at a different time. If nothing has changed, then the phase measured for each pixel will be identical to that measured previously. On the other hand, if the distance between the ground and the satellite changes between the times, perhaps due to an earthquake, then the phase measured at the satellite will change. By creating images of these phase changes, it is possible to map deformation with a precision of a small fraction of the radar wavelength: a few millimetres for the $5.6 \mathrm{~cm}$ wavelength of ERS-1 and ERS-2. In practice, the two radar images are unlikely to have been acquired from exactly the same position, introducing additional phase shifts due to the orbital separation and surface topography. $\dagger$ Using an elevation model of the target area and precise orbital models, most of these phase signals can be removed, leaving those caused by surface deformation.

Figure 5 shows an interferogram of northwest Turkey, constructed from two ERS-2 radar images acquired 35 days apart, before and after the Izmit earthquake. Each of the coloured interference fringes is equivalent to a $28 \mathrm{~mm}$ contour (half the ERS wavelength) of surface deformation in the satellite's line of sight (LOS). The phase measurements are relative, so to calculate the deformation at the fault one simply counts the fringes from the edges of the interferogram to the fault at the centre (approximately 25 on each side) and multiply by $28 \mathrm{~mm}$. In this case, the area immediately south of the fault moved more than $70 \mathrm{~cm}$ closer to the satellite and the area to the north moved by a similar amount, but away from the satellite. Knowing that the LOS of ERS-2 is nearly along the fault, but $23^{\circ}$ from the vertical, and that this earthquake produced largely horizontal motion, the $1.4 \mathrm{~m}$ LOS offset implies a horizontal fault offset of $c a .4 \mathrm{~m}$, exactly what was observed in the field.

Because none of the current crop of InSAR satellites was designed for interferometry, the fact that we can do it at all is remarkable. The technique is a fortuitous byproduct; hence, current satellite design is not optimized for InSAR, and it is not always the first priority of the mission planners. Images are not acquired as often as we would like, and satellite orbits are generally not sufficiently well steered.

Furthermore, InSAR is only possible if the character of the ground surface does not change between image acquisitions. Otherwise, there is a change in the random phase contribution due to the interaction between the radar waves and the ground, resulting in a meaningless phase change measurement (termed incoherence). Bare rock and man-made structures often remain coherent for long periods of time, but C-band (wavelength ca. $6 \mathrm{~cm}$ ) interferograms of forested areas, for example, can be incoherent even if the images were only acquired a day apart. $f$

Also troublesome is the Earth's changing atmosphere: water vapour concentrations, in particular, distort the phase ruler, causing phase shifts that can be confused with deformation. A thundercloud, for example, can cause phase changes equivalent to ground motions of up to $10 \mathrm{~cm}$. In addition, InSAR can only measure surface deformation in the LOS of the satellite. The true, three-dimensional (3D) character of the motion is lost, and we only have information from a single dimension. This can cause ambiguities in our interpretation of interferograms that lead to uncertainties in physical models. Despite these limitations, the current radar satellites, ERS-1 and

$\dagger$ By using one antenna on the Shuttle and another at the end of a $60 \mathrm{~m}$ long retractable boom, NASA's and NIMA's Shuttle Radar Topography Mission collected topographic interferograms for $80 \%$ of the Earth's land surface, taking just 10 days in February 2000. These data are currently being processed and will fill the void in high-resolution global topographic data.

$\ddagger$ This is due to multiple scattering off leaves and small branches whose positions are unstable. 
ERS-2 in particular, have produced many important observations of the earthquake cycle.

Although this paper covers the application of satellite radar interferometry to the earthquake cycle, the other major achievements of InSAR cannot go without mention. The most closely linked application is to volcanic hazards. InSAR has imaged volcanic eruptions, the inflation of volcanoes as new magma fills buried magma chambers and even the thermal contraction of erupted lava flows. Land subsidence due to mining, water extraction and oil wells has also been measured remotely using InSAR, along with landslides and even Antarctic ice flows. For more comprehensive information on how InSAR works and details of these other applications, refer to the excellent review papers by Massonnet \& Feigl (1998) and Bürgmann et al. (2000).

\section{Coseismic deformation: images of earthquakes}

For most of the 20th century, seismology provided the only way of studying the majority of continental earthquakes. By listening to the seismic waves emanating from an earthquake, seismologists can determine the location, magnitude and type of earthquake that occurred. However, there are often large uncertainties in some of the earthquake parameters for shallow crustal earthquakes: the depth of faulting is often poorly resolved with seismology and, except for very large earthquakes, the distribution of slip on the fault cannot be reliably determined. The 1992 Landers (California) earthquake furnished the first image of an earthquake's deformation field, and it graced the front cover of Nature (Massonnet et al. 1993). Since then, InSAR has been used to map the deformation resulting from nearly 30 earthquakes. This may not sound like a large number, but conventional surveying techniques had captured the deformation of less than 15 earthquakes before 1992 .

The location, magnitude and type of an earthquake can be determined from its deformation field by posing the question 'what type of earthquake could have resulted in this deformation?'. This is a classic geophysical inverse problem. Given knowledge of the earthquake fault orientation and distribution of slip, it is straightforward to calculate the deformation that we would expect to observe. Doing the reverse is difficult and computer intensive, and methods for doing this are still evolving. Nevertheless, InSAR is gradually breaking seismology's earthquake monopoly, in many cases providing vital information that was not available from seismology.

The Izmit earthquake showed how important InSAR has become. A third of the surface rupture of this magnitude 7.5 earthquake was offshore in the Gulf of Izmit, and field geologists were unable to quantify the magnitude of slip that occurred there (figure 5). Not only this, they concluded that the earthquake had terminated east of the prominent Hersek Delta, which crosses most of the Gulf, $35 \mathrm{~km}$ west of Izmit (e.g. Barka 1999). InSAR data showed that over $1 \mathrm{~m}$ of slip must have occurred beyond the Hersek delta for at least $10 \mathrm{~km}$ (Wright et al. 2001b).

The location of this termination is a crucial variable for determining the future seismic hazard for the Istanbul area. The Izmit earthquake was the seventh in a westward sequence of earthquakes that have unzipped over $1000 \mathrm{~km}$ of the North Anatolian Fault since 1939, in a relentless march towards Istanbul. The last major earthquakes on the North Anatolian Fault south of Istanbul were in 1509 and 1766 (Ambraseys \& Jackson 2000), leaving a ca. $160 \mathrm{~km}$ long seismic gap and a potential disaster on an even bigger scale than that of 1999 . 


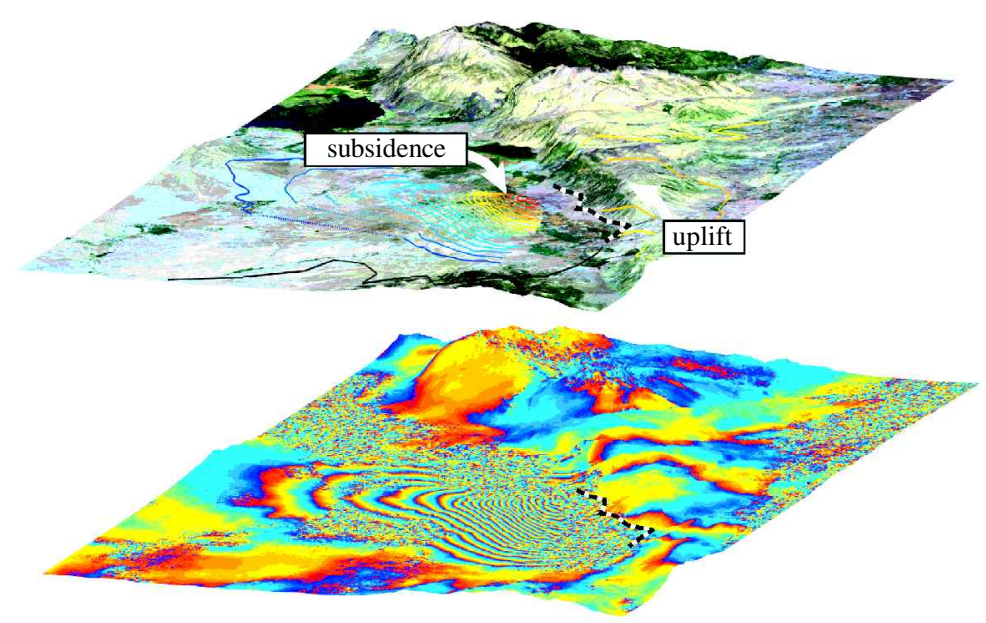

Figure 6. Two 3D views looking NW along the Dinar fault. Top, optical Landsat image draped over a digital topographic model of the area. The 1995 earthquake ruptured a $10 \mathrm{~km}$ section of the Dinar fault, marked by the black and white line, causing the area to the SW (left) to subside and the area to the NE (right) to uplift. The thin coloured lines show $28 \mathrm{~mm}$ contours of surface displacement during the earthquake, taken from the interferogram. Bottom, the Dinar interferogram. The 21 fringes $(60 \mathrm{~cm})$ of downthrow observed are eight times smaller than the three fringes $(c a .8 \mathrm{~cm})$ of uplift.

Tom Parsons and colleagues at the US Geological Survey calculated the probability of strong shaking in the Istanbul area after the Izmit earthquake. They required a historical record of earthquakes in the region and an accurate slip model of the Izmit earthquake. The latter was important because the Izmit earthquake provided an additional push to the areas at either end of the fault, increasing the seismic hazard there. Using InSAR data alone, we quickly produced a preliminary slip model. When combined with the long gap since the last earthquake, Parsons et al. (2000) estimated a $62 \%$ chance of strong shaking for greater Istanbul in the next 30 years: one of the highest probabilities for any fault zone in the world.

The earthquake that struck the town of Dinar in southwest Turkey in the autumn of 1995 was much smaller than the Izmit earthquake, but it provided direct evidence of the way that earthquakes shape the landscape. It was in an area of Turkey that is being stretched apart, and this resulted in slip on a so-called normal fault. These are faults that dip between $30^{\circ}$ and $60^{\circ}$ from horizontal and slip in such a way that one side moves up and the other side moves down during the earthquake. Using interferometry, it has been possible to image this uplift and subsidence, and relate that to the existing surface topography (figure 6) (Wright et al. 1999).

The Dinar fault is expressed geomorphologically as a sharp-fronted hill with around $700 \mathrm{~m}$ of relief across it in the area of the earthquake. During the earthquake, the higher side of the fault was jacked up by a further $7 \mathrm{~cm}$ or so, and the lower side of the fault subsided by $61 \mathrm{~cm}$. The asymmetrical uplift-downthrow ratio (ca.1:9 in this case) agrees with the predictions of simple elastic theory, but it throws up a puzzle. In the long term, geological studies of normal faults have shown that the uplift-downthrow ratio is more like $1: 2$ or $1: 3$, and yet this uplift and subsidence have to be caused by the sum of the deformation of many earthquakes. To reconcile 


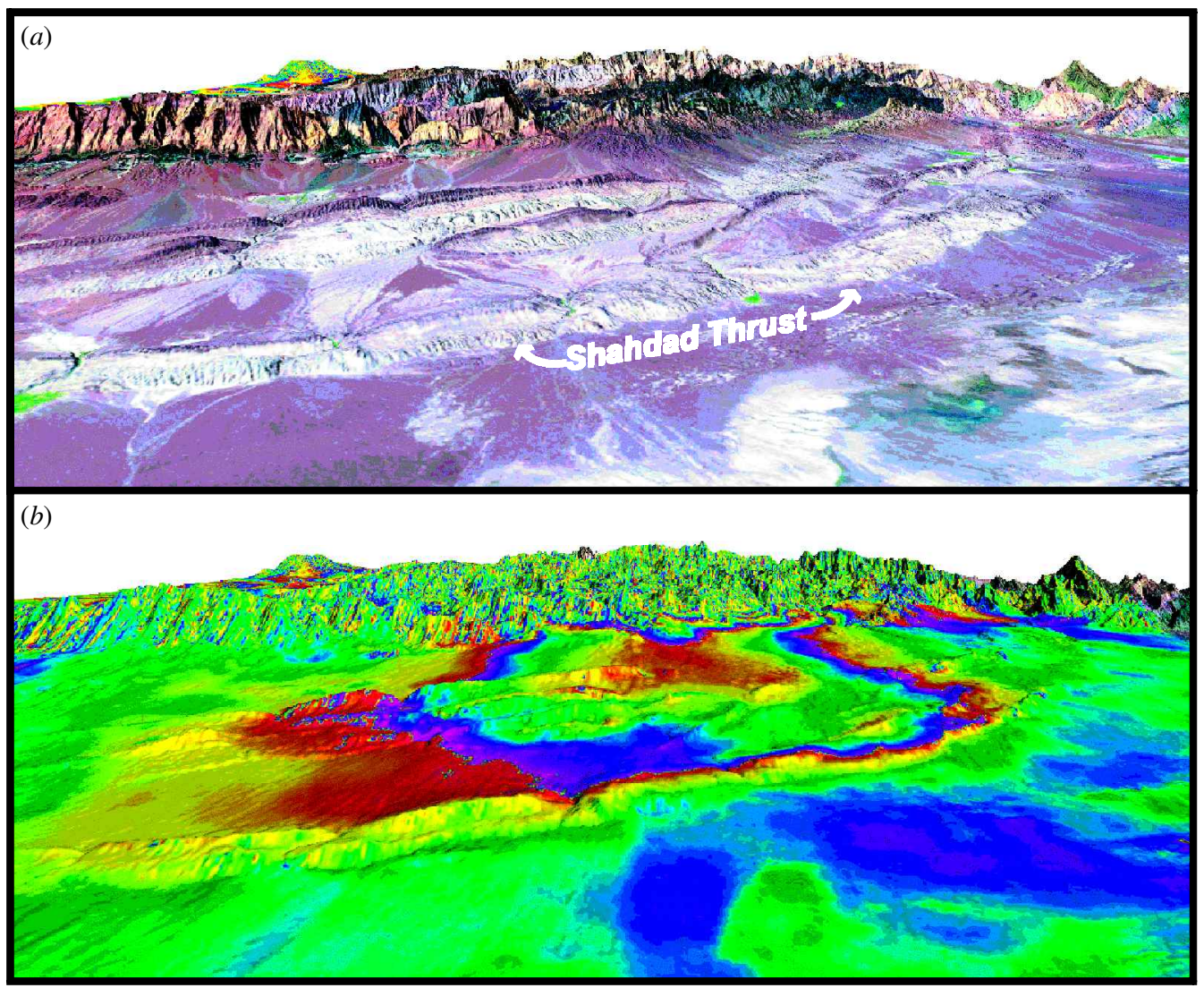

Figure 7. Perspective views of $(a)$ landscape and $(b)$ interferogram, looking west across the Shahdad Thrust (southeast Iran) towards the Abbarik Mountains, where the main fault that slipped during the 1998 Fandoqa earthquake was located (adapted from Berberian et al. 2001). The coloured interference fringe strongly associated spatially with the Shahdad Thrust shows that this structure, which dips gently away from us, must have moved by around $8 \mathrm{~cm}$ towards us, probably triggered by the earthquake in the Abbarik mountains.

this discrepancy requires postseismic relaxation, the result of aseismic ductile flow in the lower crust and/or upper mantle. Paradoxically, therefore, the confirmation of simple elastic theory in the coseismic Dinar interferogram provides compelling evidence that postseismic deformation, resulting from flowing rocks at depth, must be an important process in the earthquake cycle.

\section{Triggered slip}

Triggered slip is an example of a phenomenon that is extremely difficult to measure using conventional technology, but simple to detect with InSAR. It is defined as slip occurring during an earthquake on a fault or faults not involved in the mainshock. For example, Allen et al. (1972) observed surface cracking consistent with 10-15 mm of slip on the Imperial, Superstition Hills and San Andreas faults in California after the 1968 Borrego Mountain earthquake, a distance of 45-70 km away. Bodin et al. (1994) recorded triggered slip after the 1992 Landers earthquake on southern Califor- 
nia faults using digital creep meters that directly measure small movements across a fault. In these cases, slip began within one minute of the main shock, lasting for timeintervals of between a few hours and several weeks. At this slow speed, triggered-slip events do not produce any seismic waves and so cannot be detected with seismology. The deformation is typically small and localized, so ground-based surveying techniques can only detect it if they have a dense network spanning a fault that has moved. Digital creep meters are rarely installed on faults outside California. As a result, triggered slip was rarely observed prior to InSAR.

InSAR, on the other hand, is ideally suited to observing triggered slip because of its high spatial resolution, high measurement precision and because of the large geographical area covered by an interferogram. Indeed, InSAR has now detected many instances of triggered slip (e.g. Massonnet et al. 1994; Price \& Sandwell 1998; Wright et al. 2001b). Perhaps the most spectacular example, and one which illustrates the truly global nature of the interferometry, is that of the magnitude 6.6 Fandoqa earthquake (Iran, 1998). In addition to slip on the main, steeply dipping fault, InSAR showed that around $8 \mathrm{~cm}$ of triggered slip occurred on a shallow thrust fault, the Shahdad Thrust, over an area of some $800 \mathrm{~km}^{2}$ and in a direction nearly perpendicular to that of slip on the main fault (figure 7) (Berberian et al. 2000). This would have gone undetected without InSAR.

\section{Interseismic deformation}

Earthquakes cannot occur without the build-up of elastic strain. It is in mapping the accumulation of this interseismic strain that InSAR offers the most potential as a medium-range forecast tool. Note that I carefully avoided the term prediction, as this implies something like a two-day earthquake warning. An earthquake forecast would give the likelihood of an earthquake occurring over a certain time period (for example, 'there is a $62 \%$ chance of strong shaking in greater Istanbul in the next 30 years'). These medium-range forecasts are vital because they enable civil defence agencies to prepare communities through education, rebuilding and retrofitting programmes.

Measuring the build-up of elastic strain between earthquakes (interseismic strain) using InSAR is not straightforward. The strain rates are extremely small - the North Anatolian Fault, for example, moves at around $24 \mathrm{~mm} \mathrm{yr}^{-1}$ horizontally, and it therefore takes around three years to create a single interference fringe. Interseismic fault creep, where slip continues to the surface, produces a discontinuity that is relatively straightforward to observe in interferograms (e.g. Rosen et al. 1998). In contrast, interseismic deformation associated with faults that are locked at the surface is typically distributed on a length-scale of 30-150 km and therefore much harder to distinguish from atmospheric and orbital errors.

In an ideal world, we would look at interferograms spanning a very long timeinterval, but, in areas such as Turkey, interferograms with time-intervals of larger than two years are generally incoherent. We are therefore restricted to shorter period interferograms, but these contain such a small deformation signal that they tend to be swamped by noise. Overcoming this problem requires the use of multiple interferograms to amplify the tectonic signal and reduce the noise. By summing the cleanest four of 12 interferograms, spanning intervals between one and two years, I was able to extract the pattern of strain accumulation across the North Anatolian Fault (figure 8) (Wright 2000; Wright et al. 2001a). The stacked interferogram is effectively an 
image of the gradual build-up of elastic energy in a $c a .70 \mathrm{~km}$ wide zone across the North Anatolian Fault. This energy will eventually be released in an earthquake. The image is also a direct observation of plate tectonics in action, revealing the relative motion of Anatolia with respect to the Eurasian Plate.

Peltzer et al. (2001) have been able to measure interseismic deformation across the San Andreas Fault Zone and Mojave Desert in southern California, revealing slip rates that seem to contradict the long-term motion in some cases. The combination of field geology and interferometry suggests that the earthquake cycle in this area is complex, containing many interacting faults, possibly involved in an oscillatory pattern of strain accumulation. Current observations do not enable us to say if this is a common occurrence.

Conditions at the east end of the North Anatolian Fault and in southern California are optimal for using InSAR to determine interseismic strain accumulation: relatively large rates, reasonable coherence over $c a .2$ years and fault orientations nearly perpendicular to the ERS satellite track. Nonetheless, the fact that interseismic strain can be isolated in these locations suggests that the same can be done elsewhere, even where the rates are lower, provided a sufficient number of suitable interferograms can be obtained. With future satellite technology, it should be possible to build-up a time-varying map of strain across the whole planet and to construct accurate medium-range earthquake forecasts.

\section{Postseismic relaxation}

Measuring the response of the Earth to the known shock of an earthquake can provide vital clues to the properties of the Earth's crust and upper mantle. The interferogram of the Dinar earthquake provided indirect evidence for the flow of the lower crust or upper mantle in the aftermath of the earthquake. However, like interseismic deformation, postseismic deformation is small, occurs slowly and is therefore hard to measure directly. Nevertheless, it has been captured on a number of occasions using ground-based surveying techniques, and more recently using InSAR.

Pollitz et al. (2001) looked at several interferograms collected during the nine months after the $M=7.3$ Hector Mine earthquake (1999, Mojave Desert, California): an earthquake with around $2 \mathrm{~m}$ of mostly horizontal surface slip. They observed a long-wavelength signal with a quadrant pattern of uplift and subsidence (figure 9). They suggested that the pattern of elastic rebound that occurred in the upper brittle crust, around $16 \mathrm{~km}$ thick, changed the state of stress in the underlying layers: areas marked 'down' in figure $9 b$ were effectively subjected to elevated pressure and those marked 'up' to reduced pressure. This pressure gradient induced the rocks in the lower layers to flow away from the areas of high pressure, creating the observed spatial distribution of uplift and subsidence. For this to be true requires the upper mantle to be two orders of magnitude less viscous than the lower crust in their model and hence able to adjust rapidly after the earthquake.

As well as upper mantle flow, earthquakes can also induce changes in pore-water pressure and in some cases the deeper sections of faults are subject to so-called afterslip. The current set of observations is too narrow for the relative importance of these processes to be determined. As with coseismic deformation, InSAR has the potential to dramatically increase the number and quality of observations of this important, and yet poorly understood, phase of the earthquake cycle. 

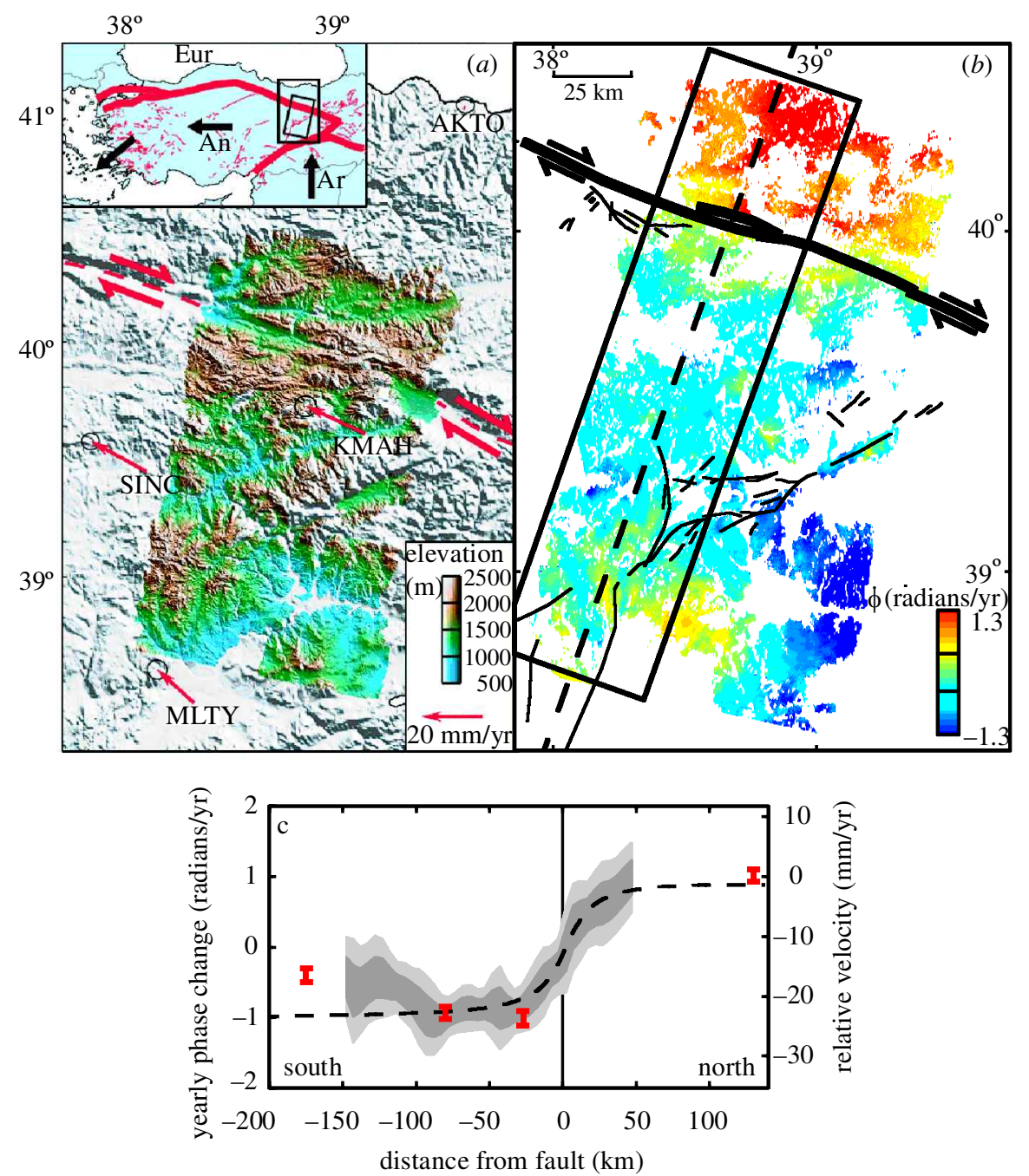

Figure 8. (a) Topographic and tectonic map of the eastern end of the North Anatolian Fault. The coloured area is an elevation model calculated from a one-day interferogram: the North Anatolian Fault (dashed red line) can clearly be seen cutting through the landscape. Arrows are GPS-determined velocities relative to the Eurasian plate (McClusky et al. 2000). (b) Stacked interseismic interferogram, converted to a yearly phase change $(\dot{\phi})$. Positive phase changes (warm colours) indicate a relative increase in distance to the satellite. (c) Phase profile perpendicular to the North Anatolian Fault (along the dashed line in $(b)$ ). The grey bands delimit the 1- and 2 -sigma error bounds with red bars the GPS velocities. Phase changes predicted by an elastic model are plotted as a dashed line.

\section{A look into the future?}

The immediate future for InSAR looks good with the recent launch of ESA's Envisat and planned launches of the Japanese ALOS satellite and Canadian Radarsat-2 in the summer of 2003. These satellites will extend the lifespan of this new technology, but none of them will greatly improve upon current capabilities. That requires something 

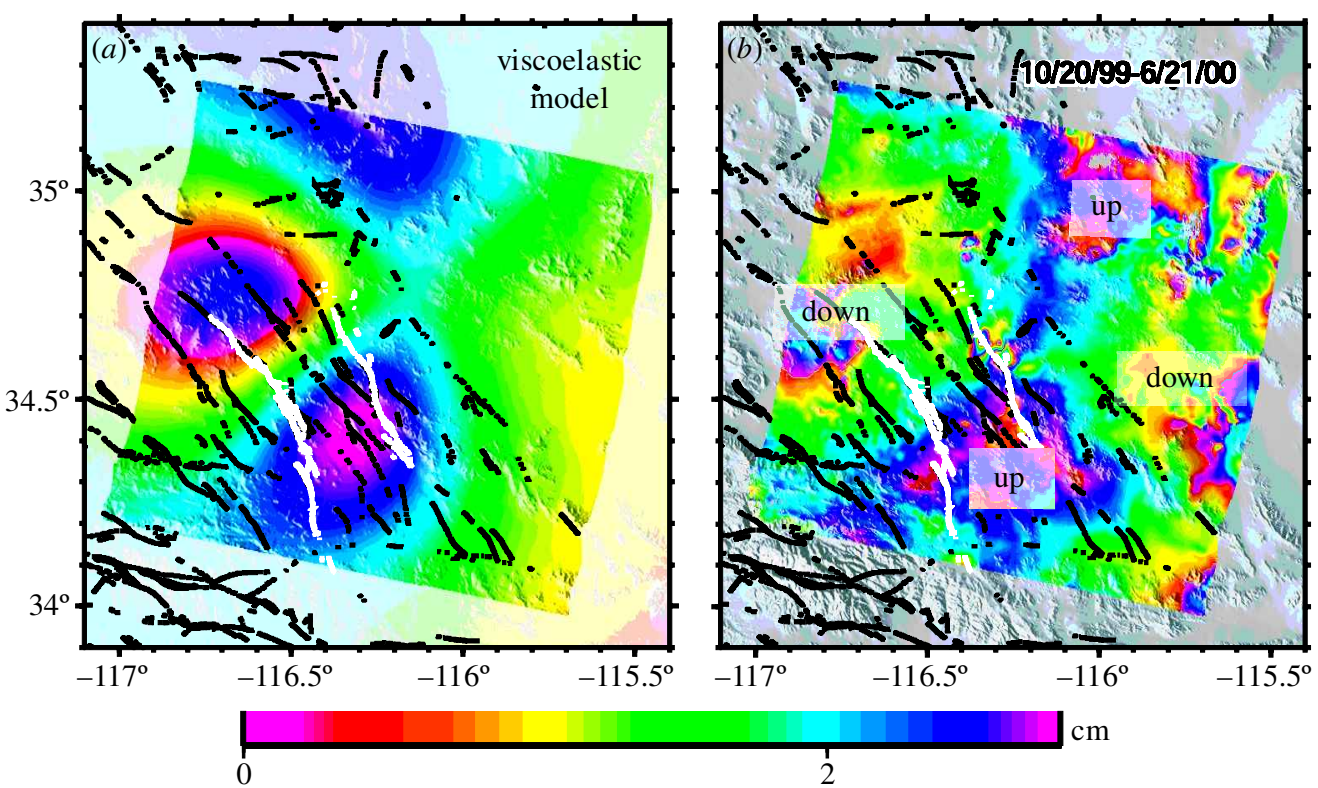

Figure 9. (a) Postseismic model and (b) interferogram for the 1999 Hector Mine interferogram (from Pollitz et al. 2001).

new: a dedicated InSAR mission, targeted at earthquake and volcanic hazards. Such a mission would have an onboard GPS to control and measure the satellite's orbit to a very high precision; it would operate at L-band $(20 \mathrm{~cm})$ wavelengths to ensure coherence is maintained, even in vegetated areas; it would collect data on every satellite pass, enabling detailed time-series to be created, atmospheric noise to be reduced, and faults with low slip rates to be monitored/identified; it would acquire data from several lines of sight, allowing 3D displacements to be recovered; and it would provide data cheaply and quickly to the scientific community. This is not a pipe dream. Two such missions, EVINSAR and ECHO, have been recently proposed to the ESA and NASA, respectively, and prospects are reasonable for the launch of at least one of these missions within the next five years.

Many of the last decade's significant earthquakes occurred on faults that had not previously been recognized as major faults of their regions (e.g. Northridge, California, 1994; Kobe, Japan, 1995; Athens, Greece, 1999). It is here that InSAR could have a big impact, by mapping strain accumulation globally and producing reliable medium-range earthquake forecasts. The human argument for such a mission is compelling: a tenth of the world's population lives in areas classified by the Global Seismic Hazard Assessment Program as having a medium-to-high seismic hazard.

The economic argument is also simple. The 1994 Northridge earthquake in Los Angeles caused total property damage estimated at $\$ 20$ billion. This would have been greater were it not for an intense programme of hazard mitigation activities over the previous two decades. Rebuilding or retrofitting structures to protect them from earthquakes is relatively cheap compared with the cost of rebuilding after an earthquake. For example, the US Federal Emergency Management Agency estimate the cost of retrofitting bridges to be just $22 \%$ of the cost if they are destroyed by earthquakes, and this does not take into account the cost to the local economy of the 
temporary loss of infrastructure. Although the cost of a dedicated InSAR mission is high (ca.\$150-250 million), a city saved from extensive earthquake damage after an InSAR forecast led to a major retrofitting programme might consider the price tag cheap. A satellite-based system is also much cheaper than attempting to make similar measurements using ground-based techniques such as continuous GPS. $\dagger$

A dedicated InSAR mission in the next 10 years, and perhaps a constellation of Earth monitoring satellites in the next 25 years, will lead to a vastly improved understanding of the physics of the earthquake cycle, a complete time-varying map of the Earth's strain and reliable earthquake forecasts that will save lives.

T.J.W. is supported by a NERC research fellowship. Thanks to Aykut Barka, Eric Fielding and Fred Pollitz for providing figures and to Barry Parsons, Jenefer Brett and an anonymous reviewer for comments that helped improve the manuscript. Apologies to those whose work could not be included in this brief review of a rapidly expanding field.

\section{References}

Allen, C., Wyss, M., Brune, J., Granz, A. \& Wallace, R. 1972 Displacements on the Imperial, Superstition Hills, and San Andreas faults triggered by the Borrego Mountain Earthquake: the Borrego Mountain Earthquake. USGS Prof. Pap. 787, 87-104.

Ambraseys, N. \& Jackson, J. 2000 Seismicity of the Sea of Marmara (Turkey) since 1500. Geophys. J. Int. 141, 1-6.

Armijo, R., Meyer, B., Hubert, A. \& Barka, A. 1999 Westward propagation of the North Anatolian fault into the northern Aegean: timing and kinematics. Geology 27, 267-270.

Barka, A. 1999 The 17 August 1999 Izmit earthquake. Science 285, 1858-1859.

Berberian, M., Baker, C., Fielding, E., Jackson, J. A., Parsons, B. E., Priestley, K., Qorashi, M., Talebian, M., Walker, R. \& Wright, T. J. 2001 The 14 March 1998 Fandoqa earthquake $\left(M_{w}\right.$ 6.6) in Kerman province, SE Iran: re-rupture of the 1981 Sirch earthquake fault, triggering of slip on adjacent thrusts, and the active tectonics of the Gowk fault zone. Geophys. J. Int. 146, 371-398.

Bodin, P., Bilham, R., Behr, J., Gomberg, J. \& Hudnut, K. W. 1994 Slip triggered on Southern California Faults by the 1992 Joshua Tree, Landers and Big Bear earthquakes. Bull. Seism. Soc. Am. 84, 806-816.

Bürgmann, R., Rosen, P. \& Fielding, E. 2000 Synthetic aperture radar interferometry to measure Earth's surface topography and its deformation. A. Rev. Earth Planet. Sci. 28, 169-209.

McClusky, S. (and 27 others) 2000 Global Positioning System constraints on plate kinematics and dynamics in the eastern Mediterranean and Caucasus. J. Geophys. Res. 105, 5695-5719.

Massonnet, D. \& Feigl, K. L. 1998 Radar Interferometry and its application to changes in the Earth's surface. Rev. Geophys. 36, 441-500.

Massonnet, D., Rossi, M., Carmona, C., Adragna, F., Peltzer, G., Feigl, K. \& Rabaute, T. 1993 The displacement field of the Landers earthquake mapped by radar interferometry. Nature 364, 138-142.

Massonnet, D., Feigl, K., Rossi, M. \& Adragna, F. 1994 Radar interferometric mapping of deformation in the year after the Landers earthquake. Nature 369, 227-230.

Parsons, T., Toda, S., Stein, R., Barka, A. \& Dieterich, J. 2000 Heightened odds of large earthquakes near Istanbul: an interaction-based probability calculation. Science 228, 661-665.

Peltzer, G., Crampé, F., Hensley, S. \& Rosen, P. 2001 Transient strain accumulation and fault interaction in the Eastern California shear zone. Geology 29, 975-978.

$\dagger$ Covering just the populated areas of the planet at risk from earthquakes with continuous GPS instruments spaced on a $15 \mathrm{~km}$ grid would cost ca. $\$ 1$ billion. 
Pollitz, F., Wicks, C. \& Thatcher, W. 2001 Mantle flow beneath a continental strike-slip fault: postseismic deformation after the 1999 Hector Mine earthquake. Science 293, 1814-1818.

Price, E. \& Sandwell, D. 1998 Small-scale deformations associated with the 1992 Landers, California earthquake mapped by synthetic aperture radar interferometry phase gradients. $J$. Geophys. Res. 103, $27001-27016$.

Reid, H. F. 1910 The mechanics of the earthquake: the California earthquake of 18 April, 1906. Report of the State Earthquake Investigation Commission, no. 2. Washington: Carnegie Institution.

Rosen, P., Werner, C., Fielding, E., Hensley, S., Buckley, S. \& Vincent, P. 1998 A seismic creep along the San Andreas Fault northwest of Parkfield, CA, measured by radar interferometry. J. Geophys. Res. 25, 825-828.

Scholz, C. H. 1990 The mechanics of earthquakes and faulting. Cambridge University Press.

Wright, T. J. 2000 Crustal deformation in Turkey from synthetic aperture radar interferometry. DPhil thesis, University of Oxford, UK.

Wright, T. J., Parsons, B., Jackson, J., Haynes, M., Fielding, E., England, P. \& Clarke, P. 1999 Source parameters of the 1 October 1995 Dinar (Turkey) earthquake from SAR interferometry and seismic bodywave modelling. Earth Planet. Sci. Lett. 172, 23-37.

Wright, T. J., Parsons, B. E. \& Fielding, E. J. 2001a Measurement of interseismic strain accumulation across the North Anatolian Fault by satellite radar interferometry. Geophys. Res. Lett. 28, 2117-2120.

Wright, T. J., Fielding, E. J. \& Parsons, B. E. $2001 b$ Triggered slip: observations of the 17 August 1999 Izmit (Turkey) earthquake using radar interferometry. Geophys. Res. Lett. 28, 10791082 . 


\section{AUTHOR PROFILE}

\section{T. J. Wright}

Born in 1974, Tim Wright graduated from Cambridge University in 1995 with first class honours in natural sciences, having specialized in Earth sciences and, to a lesser extent, physics. After spending a year working in a day centre for adults with learning difficulties, he returned to university, obtaining an MSc in remote sensing from the University of London (intercollegiate) in 1997. From 1997 to 2000 he completed his DPhil at Oxford University on InSAR studies of active tectonics in Turkey. Having completed a year's postdoctoral study at Oxford, he was then awarded a NERC postdoctoral research fellowship, also at Oxford, to study continental shear zones.

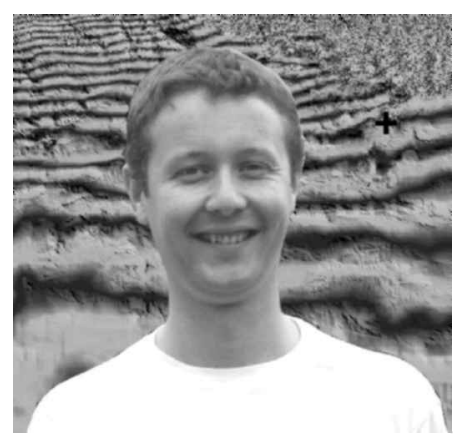

